\title{
Density Tagging Velocimetry
}

\author{
Markus Raffel - Ricardo Hernandez-Rivera - Benjamin Heine - Andreas \\ Schröder · Karen Mulleners
}

Received: date / Accepted: date

\begin{abstract}
Density Tagging Velocimetry, a novel optical technique for point-wise measurement of flow velocity is proposed here. This new method is based on the detection and subsequent tracking of a local density variation deliberately inserted in the flow. The experimental implementation comprising tagging, detection, and velocity evalution reverts to and combines principles of well-known optical measurement techniques. Density Tagging Velocimetry has the potential for inflight application and is particularly suited for measuring flow velocities in regions where the use of tracer particles is difficult or undesired. The applicability of this new technique is illustrated by a jet flow measurement.
\end{abstract}

\section{Introduction}

Most common flow velocity measurement techniques in modern experimental fluid dynamics are optical methods that rely on the imaging and tracking of particles which have been added to the fluid-flow, e.g. Particle Image Velocimetry (PIV), Laser Doppler Velocimetry (LDV), and Light Detection and Ranging (LiDaR). The main advantage of these optical techniques compared to conventional velocity probes, e.g. hot wires and Pitot tubes, is their non-intrusiveness. However, finding adequate tracer particles that accurately follow the flow is challenging. Occasionally, we encounter situations where the use of tracer particles is difficult (e.g. in specialised wind tunnels or microchannels and during

M. Raffel ( ) · R. Hernandez-Rivera · B. Heine · A. Schröder K. Mulleners

German Aerospace Center (DLR),

Bunsenstr. 10, 37073 Göttingen, Germany

E-mail: markus.raffel@dlr.de in-flight measurements) or can lead to complications (e.g. in combustion engines, high pressure vessels, or flames). Low tracer densities in vortex cores or within boundary layers hamper accurate flow velocity quantification and alternative techniques are desirable.

Hitherto, several techniques have been proposed that do not require tracer particles to be added to the flow. Image Correlation Velocimetry (Tokumaru and Dimotakis 1995) and Schlieren "PIV" (Jonassen et al 2006) use the prevalent eddies in a turbulent flow as Lagrangian flow tracers. Due to the need for naturally occurring density gradients, the applicablity is limited to turbulent or thermal flows. Molecular Tagging Velocimetry (MTV) is a whole field optical technique based on the successive recordings of lines or grids of tagged fluid molecules (Popovich and Hummel 1967; Hiller et al 1984; Gendrich et al 1997; Tropea et al 2007). The experimental implementation is rather complicated which makes MTV inadequate for full-scale in-flight measurements.

Full-scale in-flight experiments have become increasingly indispensable since not all relevant dimensionless parameters can be attained in small-scale testing. Furthermore, they have become increasingly desired since they provide access to much larger range of flight parameters than usually attainable in wind-tunnel testing. This is accompanied by a high demand for novel experimental methods allowing to investigate velocity field characteristics in situ.

In this letter we propose a novel point-wise measurement technique that is versatile, easy to implement and does not rely on the addition of tracer particles. This new technique adopts principles from well known optical techniques such as PIV and schlieren photography and is named Density Tagging Velocimetry (DTV). In the following, the basic idea or operation principle is 
presented prior to the experimental implementation of DTV including tagging methods, detection of the density variation, and velocity evaluation. Validation of the DTV technique was provided by comparing the mean velocity profile across a cold jet with PIV data.

\section{Density Tagging Velocimetry}

\subsection{Measurement Principle}

The proposed DTV method is an optical technique for pointwise velocity measurements based on the detection and subsequent tracking of a local refractive-index or density variation which is deliberately induced in the flow. The local density variation, termed density tag, acts as a tracer particle which is transported by the fluid flow. The velocity information is obtained by determining the position of the density tag at two subsequent instances in time based on the Background Oriented Schlieren (BOS) principle. Dividing the Lagrangian displacement of the density tag by the time interval between the two recordings is a direct estimate for the local velocity vector. A standard one detector DTV set-up allows for a 1D2C velocity measurement, i.e. in a spatial point two components of the velocity vector are determined. The third velocity component can be obtained utilising a two camera system set-up.

\subsection{Experimental Implementation}

The experimental implementation comprised three stages, tagging, detection and processing. For each of the stages multiple possibilities are presented and discussed.

\subsubsection{Tagging methods}

The DTV method does not rely on naturally occurring density gradients, unlike e.g. Image Correlation Velocimetry (Tokumaru and Dimotakis 1995) and Schlieren "PIV" (Jonassen et al 2006), and the potential field of application is wide. Although the operation principle is universal, different applications ask for different tagging methods. We provide here a number of conceivable tagging schemes for individual areas of application and discuss their adequateness in terms of simplicity, feasibility, and intrusiveness.

For application to air flows, a tagging method based on laser induced ionisation or plasma is proposed. A focused pulsed laser beam yields the emergence of a plasma spark and a local density variation as a result of the high energy density in the focal region. A convex or converging lens is used to focus a collimated laser

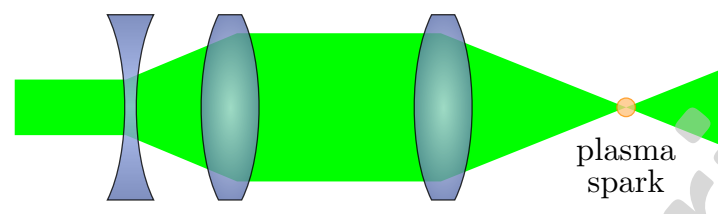

Fig. 1: Schematic representation of the optical arrangement for plasma generation consisting of a convex or converging lens preceded by a combination of a concave and a convex lens to reduce spherical aberation.

beam to a spot on the lens axis. To ensure that the light is collimated or parallel when passing through the converging lens, hence reducing spherical aberration, the beam focussing optics comprise a suitable combination of a convex and a concave lens (see figure 1). This approach is termed plasma tagging and is deemed an adequate tagging method allowing in-situ velocity measurements in inaccessible locations and hostile environments. More advantages of laser induced ionisation are:

- accurate spatial tagging yielding high spatial resolution measurements;

- generation of anisotropic density variation which facilitates detection and processing;

effective adjustment of the spot size by varying the laser intensity; and

- accurate reproducibility.

Although the plasma is induced in a non-intrusive way, i.e. without utilising intrusive probes, the ionisation spark itself alters the local air properties such as temperature and density. To what extent the generation of the plasma influences the flow velocity need further investigation.

Other conceivable tagging schemes are electric sparks, local heating of the wall in a micro-channel, and the pulsed injection of a fluid of a different material or temperature, such also helium bubbles or hot air bubbles. Again, these methods locally alter the fluid properties and the degree of intrusiveness and subsequent inaccuracy for different applications has to be scrutinised. This discussion is beyond the scope of the present letter and we postpone it to future publications.

\subsubsection{Detection}

For the detection of the externally induced density variation, we revert to the well-known optical Background Oriented Schlieren (BOS) method, also referred to as Background Oriented Optical Tomography (BOOT) or Synthetic Schlieren technique (Raffel et al 2000; Richard and Raffel 2001; Dalziel et al 2000). 

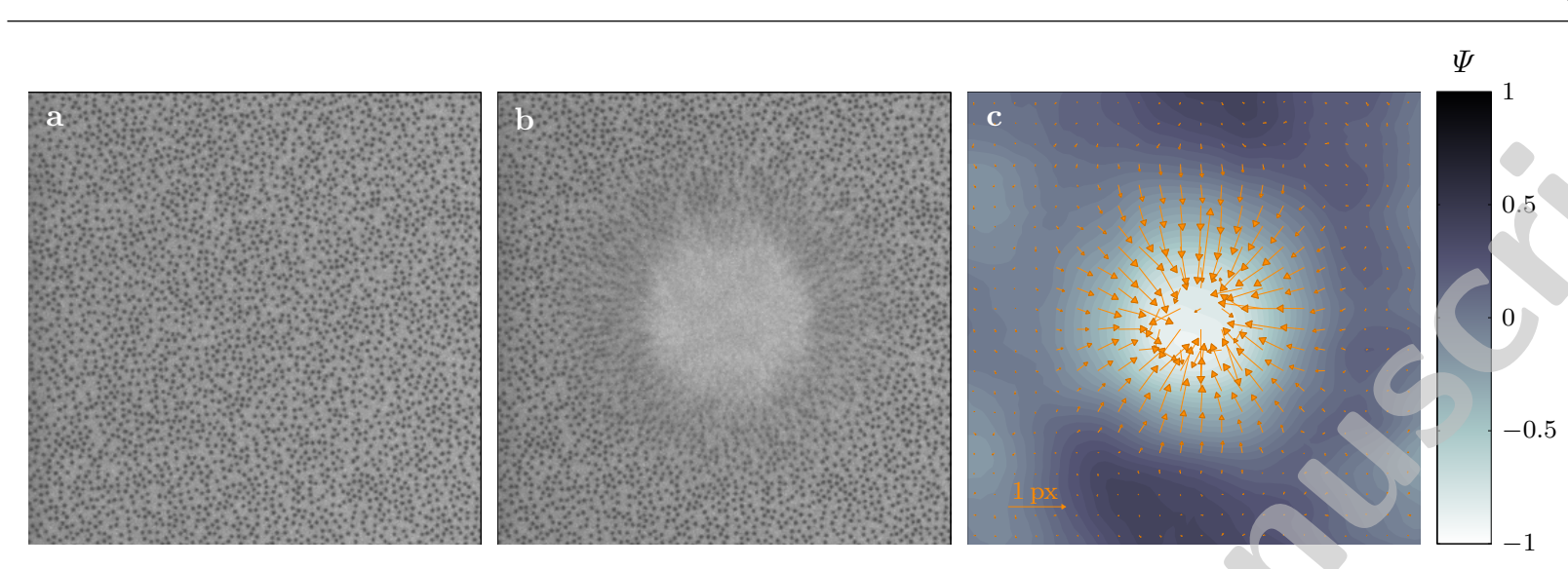

Fig. 2: Background pattern recording $\mathbf{a}$ before and $\mathbf{b}$ shortly after the density variation was brought into the flow by means of plasma tagging; c correlation between both recordings where the spatial distribution of $\Psi$ (eq. 1) is colour-coded.

The BOS technique is based on the deflection of light due to refractive index changes related to density gradients in the investigated flow. A background speckle pattern with a high spatial frequency and high contrast is recorded with and without the density variation influencing the light path. The displacement of the background pattern with respect to the reference image is evaluated by means of standard correlation methods, e.g. those included in PIV processing software.

Exemplary recordings of the background speckle pattern before and shortly, i.e. $10 \mu$ s, after a laser induced plasma spark was generated are depicted in figure $2 \mathbf{a}$ and $\mathbf{b}$, respectively. The corresponding speckle displacement vector field is presented in figure 2c. The plasma was generated with a pulsed Nd:YAG laser operated with $44 \mathrm{~mJ}$ pulse energy. A single CCD camera was utilised which was focused on the background pattern. To increase light intensity a retro-reflective speckle patterns was adopted in combination with high power, pulsed LED light sources Roesgen (2000); Heineck et al (2010).

Generally, artificial speckle patterns are used for BOS, but the applicability of natural backgrounds such as grass, the edge of a cornfield, or a tree grove, has been demonstrated (Kindler et al 2007; Hargather and Settles 2010). Hence, DTV has the potential for in-situ and in-flight measurements.

\subsubsection{Velocity Evaluation}

With regard to velocity evaluation schemes, we distinguish two different types, approaches based on the speckle displacement vector field and procedures where the correlation step is omitted.
Considering the speckle displacement vectors in figure $2 \mathbf{c}$, they are directed towards the centre of the density tag. Hence, in order to determine the precise location of the centre of the spot, a simple algorithm is proposed based on the spatial function

$$
\begin{aligned}
\Psi\left(\mathrm{x}_{\mathrm{i}}\right) & =\frac{1}{M} \sum_{\mathbf{x}_{\mathbf{j}} \in S_{\mathrm{i}}} \frac{\left[\left(\mathbf{x}_{\mathbf{j}}-\mathbf{x}_{\mathrm{i}}\right) \cdot \mathbf{d} \mathbf{x}_{\mathbf{j}}\right] \cdot \mathbf{n}}{\left|\mathbf{x}_{\mathbf{j}}-\mathbf{x}_{\mathrm{i}}\right| \cdot\left|\mathbf{d} \mathbf{x}_{\mathbf{j}}\right|} \\
& =\frac{1}{M} \sum_{\mathbf{x}_{\mathbf{j}} \in S_{\mathrm{i}}} \cos \left(\theta_{\mathrm{ij}}\right)
\end{aligned}
$$

with $S_{\mathrm{i}}$ a two-dimensional area around the spatial location $\mathbf{x}_{\mathrm{i}}, M$ the number of grid points $\mathbf{x}_{\mathrm{j}}$ inside $S_{\mathrm{i}}$ with $j \neq i, \mathbf{n}$ the unit normal vector, $\mathbf{d x}_{\mathbf{j}}$ the vector displacement at $\mathbf{x}_{\mathbf{j}}$, and $\theta_{\mathrm{ij}}$ the angle formed by the vectors $\mathbf{x}_{\mathbf{j}}-\mathbf{x}_{\mathrm{i}}$ and $\mathbf{d} \mathbf{x}_{\mathbf{j}}$. According to its definition, $\Psi$ is a dimensionless scalar function, with $-1 \leq \Psi \leq 1$. The centre position of the density spot is indicated by the local extrema of $\Psi$ (see figure $2 \mathbf{c}$ ). Sub-pixel accuracy can be obtained by fitting the dominant peak of $\Psi$ to a two-dimensional Gaussian distribution. This approach was inspired by the vortex identification scheme by Michard et al (1997).

Alternatively, the position of the spot can be determined directly form the recorded intensity images. For this purpose the reference image is subtracted from the displaced image, yielding high values in the region of the density variation and low values elsewhere. The intensity centroid of the subtraction image indicates the position of the density tag with an improved accuracy compared to the Psi-based method, even when considering small displacements, cf. Optical Flow evaluation by Horn and Schunck (1981). Omitting the correlation step also yields an important reduction in processing time. This is extremely interesting in view of real-time 


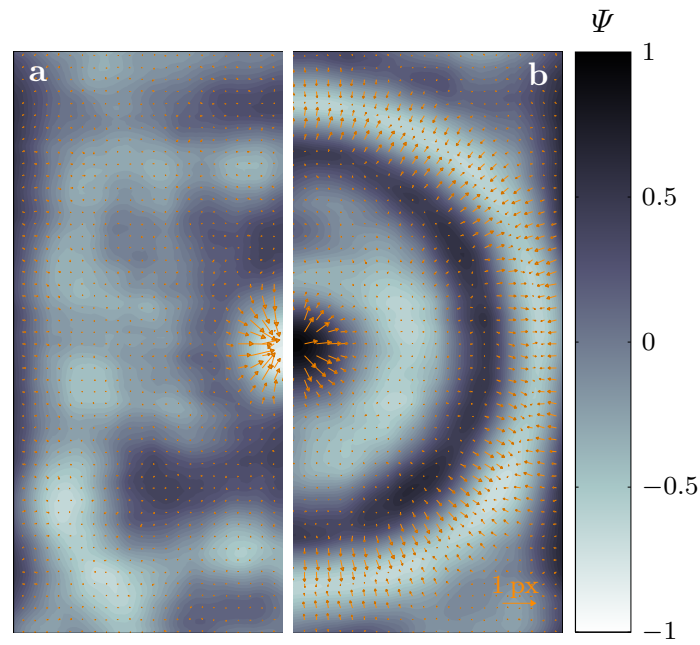

Fig. 3: Speckle displacement vector field a $10 \mu \mathrm{s}$ and b $50 \mu$ s after plasma generation, indicating the shock wave emission around the plasma spark. The corresponding $\Psi$-distribution is colour-coded.

measurements and implementation in closed loop controlling circuits. The objective of this paper is to present the DTV method and the debate on the accuracy of the different processing schemes is deemed interesting but considered future work.

\subsection{Further Considerations}

\subsubsection{Measurement Accuracy}

The spatial accuracy is determined by the size of the plasma spot, which in turn is defined by the energy density and beam diameter, i.e. lens setup, beam quality, and $\mathrm{M}^{2}$-factor. The velocity resolution on the other hand depends on the size and resolution of the camera chip, the magnification, and the time delay between the subsequent recordings, alike for PIV measurements, and on the selected evaluation scheme. The accuracy of the latter has to be scrutinised and is the subject of future investigations. Based on the authors' experience the plasma tagging method is preferably applied to high velocity flows, where the physical displacements are larger. In addition to the spatial and velocity resolution, the intrusiveness has an influence on the measurement accuracy. To what extent the induced density variations locally alters the flow field depends on the coherence of the measured flow, the magnitude of the density gradient, and the strength of the shockwave. Whereas the last two can be regulated e.g. through the laser pulse energy in case of plasma tagging, the for- mer is a given and determines the area of application of DTV.

\subsubsection{Temperature of the Gas Flow}

In addition to the velocity measurement, the plasma tagging procedure allows for a simultaneous estimate of the temperature of gas flows. Characteristic of a laser induced ionisation in air is the shock wave emission around the plasma spark (figure 3). The propagation rate of the wave fronts equals the speed of sound $c$ which, for an ideal gas, is given by

$c=\sqrt{\frac{\gamma R T}{M}}$

where $\gamma$ is the adiabatic constant of the specific gas, $M$ the molecular weight of the gas, $R$ the universal gas constant, and $T$ the absolute temparature. Hence, the shock wave propagation velocity is an estimate for the global gas temperature.

\subsubsection{Plasma Image Velocimetry (PlIV)}

In high-velocity flows, the plasma spark itself can be used as tracer particle. Consider a plasma generated by a pulsed Nd-YAG laser operated with a pulse energy of $100 \mathrm{~mJ}$. While the laser pulse width itself is of the order of 10 to $10^{2} \mathrm{~ns}$, the plasma spark continues to be visible for approximately $20 \mu \mathrm{s}$. Velocity information can then be obtained based on the position of the plasma glow instead of the density variation accompanying it. The major advantage is that the background pattern becomes redundant, which is highly desirable for in-flight measurements.

In contrast to the previously described standard DTV, the sensitivity of PIIV can not be ameliorated by increasing the delay time between the recordings since the plasma glowing is finite. Hence, PIIV is preferentially used for measurement of high-velocity flows where the displacement of the glow during its short life time is sufficiently large.

\section{Proof of Concept}

In order to demonstated the applicability of DTV, a cold jet flow was measured by means of both DTV and PIV. We focused on the laminar part of the jet flow where the velocity profile in the core of the jet is steady and uniform. These conditions are well suited for a first test of the DTV method and for comparison of DTV and PIV results. 

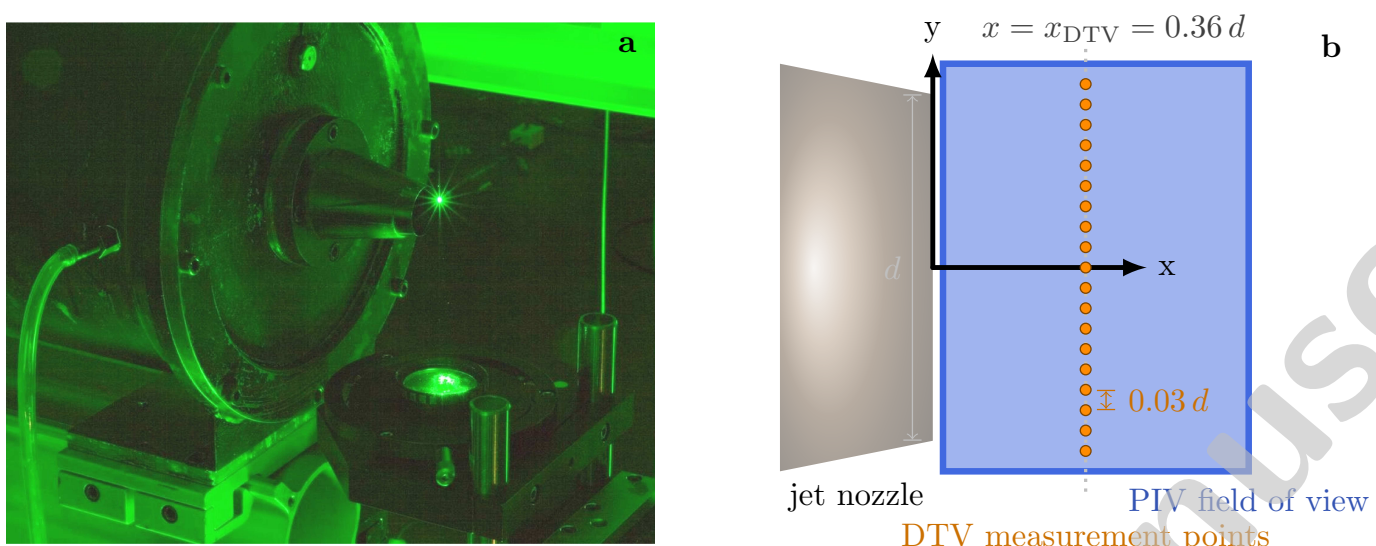

DTV measurement points

Fig. 4: Experimental set-up a photograph of the DTV configuration with ionisation spark $\mathbf{b}$ schematic position of the PIV field of view and the DTV measurement points.

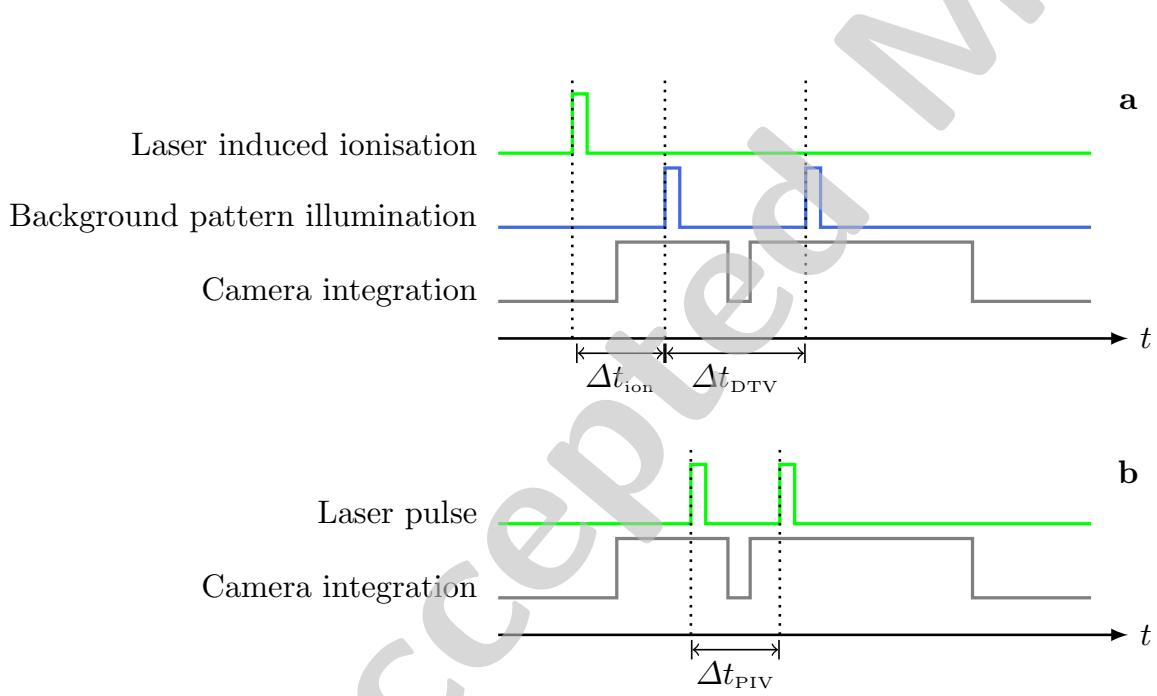

Fig. 5: Timing diagrams for a DTV and $\mathbf{b}$ PIV recording.

\subsection{Experimental Set-up}

The cold jet was generated by a circular nozzle with a diameter $d=25 \mathrm{~mm}$ mounted on a cylindrical settling chamber with a length of $0.5 \mathrm{~m}$ and a diameter of $0.2 \mathrm{~mm}$ (cf. Henning et al 2010). The mean exhaust velocity of the jet was regulated by adjusting the pressure at rest. With the jet flow directed horizontally, the velocity in the vertical symmetry plane directly behind the nozzle's exit was measured by means of both DTV and PIV.

Concerning DTV, laser induced plasma tagging was adopted to generate a density variation (section 2.2.1). For this purpose, a pulsed frequency-doubled Nd-YAG laser operating at a repetition rate of $10 \mathrm{~Hz}$ with a pulse energy of $44 \mathrm{~mJ}$ was utilised. By focussing the laser beam, an ionisation spark was generated at a defined location within the region of interest. The plasma tag was traversed across the jet flow to obtain average velocity profiles (figure 4).

The density variation was detected utilising a standard BOS setup consisting of CCD camera with doubleshutter mode and a retro-reflective background pattern. To obtain velocity information, two subsequent snapshots, i.e. recordings with a very short exposure time, of the density tag were required. Since the camera exposure time could not be set arbitrarily short, we applied pulsed illumination of the background pattern to obtain instantaneous recordings of the density variation, comparable to the double-pulsed laser illumination commonly used for double-frame PIV. The local flow velocity was evaluated from the frame-to- 


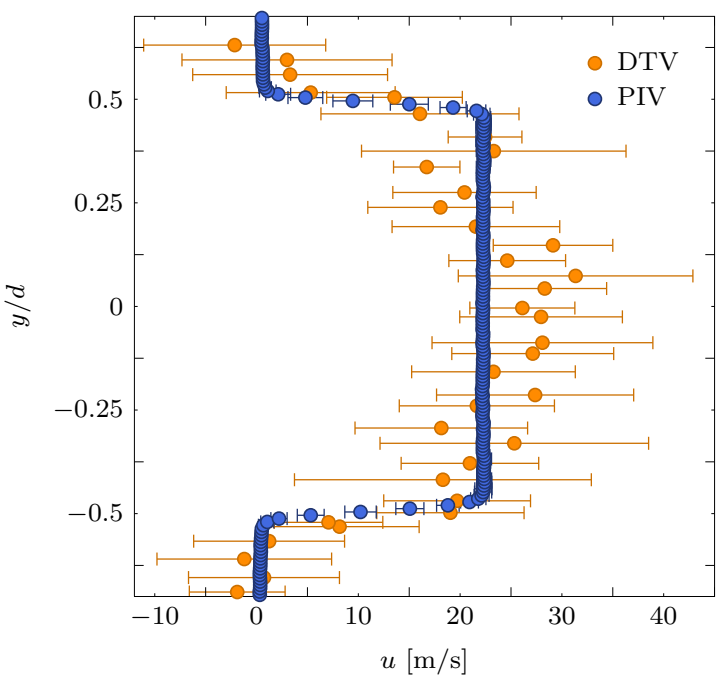

Fig. 6: Average velocity profile across the jet flow at $x=0.36 d$ obtained by means of both DTV and PIV.

frame displacement of the plasma tag, whose position was determined based on the $\Psi$-criterion described in section 2.2.3. Prior to computing $\Psi$, the mean displacement over the region of interest was subtracted to account for vibrations.

Reference velocity field data was acquired in the vertical symmetry plane directly downstream of the nozzle exit with a standard one camera planar PIV system (figure $4 \mathbf{b}$ ). The PIV data was evaluated according to standard procedures (Raffel et al 2007).

The timing of the fundamental events for the DTV and standard double frame PIV are depicted graphically in figure 5. For the present application, the interframing time between the DTV recordings was $\Delta t_{\mathrm{DTV}}=$ $40 \mu \mathrm{m}$ and the time delay between the plasma ionisation and the first recording was $\Delta t_{\text {ion }}=10 \mu \mathrm{m}$. To avoid that the very bright ionisation spark hampers the visualisation of the density variation, the camera exposure for the first recording was started shortly after the plasma was generated. The interframing time for PIV was $\Delta t_{\mathrm{PIV}}=10 \mu \mathrm{m}$.

\subsection{Comparison of DTV and PIV Results}

The streamwise mean velocity profiles at the downstream location $x_{\mathrm{DTV}}=0.36 \mathrm{~d}$ measured by means of by DTV and PIV are depicted in figure 6 . The PIV result, which is the average of 100 instantaneous vector fields, clearly shows the uniform profile characteristic of a laminar jet flow.

The uniform core velocity was estimated by averaging over the measurements points for which applies $-0.4<y / d<0.4$, yielding $22.25(4) \mathrm{m} / \mathrm{s}$ and $24(4) \mathrm{m} / \mathrm{s}$ for PIV and DTV respectively. At the edges of the jet, where the strongest velocity gradient is present, the DTV and PIV results are in good agreement.

However, the standard deviation, indicated by the errorbars, is much larger for DTV than for PIV in this example. This has multiple reasons. First, only 10 measurements per location have been taken into account. While PIV allows for velocity field measurements, DTV is a point-wise measurement technique and requires a number of measurements in different spatial locations in order to obtain an average planar velocity distribution. DTV is more time-consuming, but the use of an effective traversing system can significantly decrease the overall measurement time. Second, the pixel displacement of the plasma is small in the present case. Minor absolute errors in the determination of the position can lead to significant relative errors in the velocity. Third, the evaluation procedure chosen here is based on the image correlation and the size of the correlation windows limits the accuracy of the position and velocity evaluation.

Based on the above presented example, we demonstrated that the proposed DTV method allows for pointwise velocity measurements in gas flows even though the implementation needs further improvement. We expect that by optimising the evaluation schemes and increasing the image magnification a measurement accuracy comparable to the one obtained by standard PIV algorithms can be reached.

\section{Conclusion and Future Work}

A new optical point-wise velocity measurement technique DTV was presented. DTV is a simple and robust technique allowing for pointwise measurement of velocities where tracer particles are not practical or welcome. The experimental implementation was divided in three stages, tagging, detection, and evaluation. The most promising tagging method termed plasma tagging was based on laser induced ionisation. To detect and track the density variation we reverted to and combine well known optical techniques such a BOS and the computation of spatial correlations. This is deemed to be the stength of DTV, every laboratory equipped with a PIV laser and software can perform DTV. Although further and detailed investigation of the intrusiveness of different tagging methods, the accuracy of various velocity evaluation schemes, and the limitations of DTV is desirable, first comparison between DTV and PIV results of a cold jet flow were promising. 


\section{References}

Dalziel S, Hughes G, Sutherland B (2000) Whole-field density measurements by synthetic schlieren'. Exp Fluids 28:322-335

Gendrich C, Koochesfahani M, Nocera D (1997) Molecular tagging velocimetry and other novel applications of a new phosphorescent supramolecule. Exp Fluids 23(5):361-372

Hargather M, Settles G (2010) Natural-background-oriented schlieren imaging. Experiments in Fluids 48:59-68

Heineck J, Schairer E, Walker L, Kushner L (2010) Retroreflective background oriented schlieren (rbos). In: proceedings of the 14th Int. Symposium on Flow Visualization (ISFV14), EXCO Daegu, Korea

Henning A, Schröder A, Krebs I, Agocs J (2010) Aeroacoustic investigations on a cold jet by means of simultaneous piv and microphone measurements. In: proceedings of the 15th Int. Symposium on Applications of Laser Techniques to Fluid Mechanics, Lisbon, Portugal

Hiller B, Booman R, Hassa C, Hanson R (1984) Velocity visualization in gas flows using laser-induced phosphorescence of biacetyl. Review of Scientific Instruments 55(12):1964 -1967

Horn B, Schunck B (1981) Determining optical flow. Artificial Intelligence pp 185-203

Jonassen D, Settles G, Tronosky M (2006) Schlieren piv for turbulent flows. Optics and Lasers in Engineering 44:190-207

Kindler K, Goldhahn E, Leopold F, Raffel M (2007) Recent developments in background oriented Schlieren methods for rotor blade tip vortex measurements. Exp Fluids 43:233-240

Michard M, Graftieaux L, Lollini L, Grosjean N (1997) Identification of vortical structures by a non local criterion - application to PIV measurements and DNS-LES results of turbulent rotating flows. In: Proceedings of the $11^{\text {th }}$ Conference on Turbulent Shear Flows, Grenoble, France

Popovich AT, Hummel RL (1967) A new method for nondisturbing turbulent flow measurements very close to a wall. Chemical Engineering Science 22(1):21-25

Raffel M, Richard H, Meier G (2000) On the applicability of background oriented optical tomography for large scale aerodynamic investigations. Exp Fluids 28(5):477-481

Raffel M, Willert C, Wereley S, Kompenhans J (2007) Particle Image Velocimetry, a Practicle Guide, 2nd edn. Experimental Fluid Mechanics, Springer

Richard H, Raffel M (2001) Principle and application of the background oriented Schlieren (BOS) method. Meas Sci Technol $12: 1576$

Roesgen T (2000) private communication, zürich, Switzerland

Tokumaru P, Dimotakis P (1995) Image correlation velocimetry. Exp Fluids 19(1):1-15

Tropea C, Yarin AL, Foss JF (eds) (2007) Springer Handbook of Experimental Fluid Mechanics, Springer, chap Velocity, Vorticity and Mach number, pp 362-382 